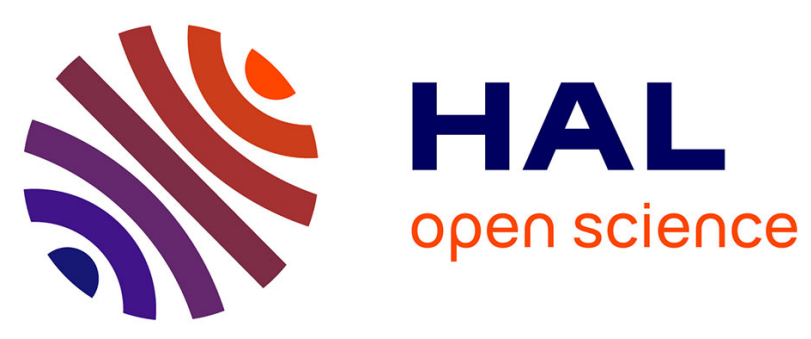

\title{
A robust nonlinear semi-active control for base seismically-isolated structures
}

Catalin-Stefan Teodorescu, Sette Diop, Ioannis Politopoulos, Messaoud Benidir

\section{- To cite this version:}

Catalin-Stefan Teodorescu, Sette Diop, Ioannis Politopoulos, Messaoud Benidir. A robust nonlinear semi-active control for base seismically-isolated structures. MED 2013 - 21st Mediterranean Conference on Control and Automation, Jun 2013, Chania, Greece. Paper 211, 10.1109/med.2013.6608775 . hal-00828890

\section{HAL Id: hal-00828890 \\ https://hal-centralesupelec.archives-ouvertes.fr/hal-00828890}

Submitted on 31 May 2013

HAL is a multi-disciplinary open access archive for the deposit and dissemination of scientific research documents, whether they are published or not. The documents may come from teaching and research institutions in France or abroad, or from public or private research centers.
L'archive ouverte pluridisciplinaire HAL, est destinée au dépôt et à la diffusion de documents scientifiques de niveau recherche, publiés ou non, émanant des établissements d'enseignement et de recherche français ou étrangers, des laboratoires publics ou privés. 


\title{
A robust nonlinear semi-active control for base seismically-isolated structures
}

\author{
C.-S. Teodorescu ${ }^{1}$, S. Diop ${ }^{2}$, I. Politopoulos ${ }^{1}$ and M. Benidir ${ }^{2}$
}

\begin{abstract}
This paper proposes a robust nonlinear semi-active control for base seismically-isolated structures. The control is based upon an extension of works of Leitmann et al. on the stabilization of nonlinear systems with uncertain models. For usual models of structure dynamics it is shown that applying a specific control law drives the state variables into a ball of specified radius in finite time. The radius of the ball may be arbitrarily chosen as long as it is not lower than a limiting value. In addition, estimates of this limiting ball radius is provided. The time to reach the ball is also provided. The semi-active control thus provides the control designer with interesting design parameters. The efficacy of proposed semiactive control is illustrated by its application to simple models of structures focusing in particular to the attenuation of excitation transmitted from floor to equipment mounted on them.
\end{abstract}

\section{INTRODUCTION}

Base isolation is an alternative solution in earthquake protection of structures and equipment. Typically, a base isolated structure is put on very flexible bearings (e.g., rubber bearings). Roughly speaking this is the socalled passive control solution. Another solution, known as active control, consists of an ideal actuator which is controlled in order to stabilize the structure in presence of seismic disturbance. Such an actuator is assumed to be able to produce as well as dissipate energy in order to reduce structure motion. Typical active control actuators are the controlled hydraulic cylinders. On the other hand, the so-called semi-active control, is implemented through actuators that can only dissipate energy. Contrary to passive control devices the semi-active control actuators, typically, are viscous dampers whose parameters can be controlled by low power means. Passive control devices are fixed once for all while active control devices require too much energy. This is to say that semi-active control actuators are interesting alternative solutions for the protection of buildings and structures. In this work we consider actuators which consist of passive and semi-active devices. When designing active control laws

\footnotetext{
${ }^{1}$ CEA, DEN, DANS, DM2S, SEMT, Laboratoire d'Etudes de Mécanique Sismique, F-91191 Gifsur-Yvette, France, Catalin-Stefan Teodorescu <catalin-stefan.teodorescu@cea.fr>, Ioannis

Politopoulos <ioannis.politopoulos@cea.fr>

${ }^{2}$ Laboratoire des Signaux \& Systèmes, CNRS Université Paris-Sud Orsay, SUPELEC, Plateau de Moulon, F-91192 Gif-sur-Yvette, France, Sette Diop <sette.diop@lss.supelec.fr>, Messaoud Benidir

the structure dynamics often may be assumed linear, making the theoretical synthesis much easier while the real implementation is quite impossible. On the other hand semi-active control design is basically nonlinear while its implementation is being considered in near future installations. The semi-active control design is more involved compared to the active control design.

Most of previous works used a two-step procedure: a reference is first calculated using an active control law then this reference is approximated at the best utilizing available devices. The reader may refer to Politopoulos and Pham [14] where LQR techniques are used to calculate the above mentioned reference.Precisely, the initial motivation of this work was to design a semi-active control law for the two-degree-of-freedom structure of the latter paper. This is done by extending some work by Leitmann and co-authors [9]. In [9] a nonlinear active control is presented. In the present paper a nonlinear semi-active control strategy is proposed.

In addition to the requirement of satisfactory behavior of main structures (e.g., buildings), for industrial facilities an important issue is the proper functioning of equipment during and after an earthquake. To this end, pseudoacceleration floor response spectra which will be defined in Section III give relevant information. The main objective of this control design is to keep these floor spectra as low as possible. However the floor response spectrum is not an enough explicit criterion for control design techniques.

Most previous works use performance criterion in terms of maximum values of relative or absolute coordinates. In this paper a tentative use of the floor response spectrum is undergone. This is done by having recourse to modal coordinates. Penalties are introduced on modal coordinates that are responsible for generating higher values on the floor response spectrum.

The paper is organized as follows: The model of the structure is presented in the next section. In Section III we provide an account on the definition of floor response spectrum which shows the difficulty of using this quantity as a control design criterion. Section IV is devoted to the stabilization theory of nonlinear systems with uncertain models stemming from works of Leitmann and co-workers. Our extension of one of their results is used in Section $\mathrm{V}$ to design the semi-active control 
which is proposed in this work. Finally simulations are shown illustrating the performance of this control law. Improvements over passive control will be seen as a noticeable result.

\section{Plant MODEL}

The model of base isolated structures that is considered in this work is the following mass-spring-damperfriction dynamic equations

$$
\begin{aligned}
& M \ddot{z}_{\mathrm{a}}+\left(C+C_{\mathrm{c}}\left(c_{\mathrm{A}}\right)\right)\left(\dot{z}_{\mathrm{a}}-1_{n \times 1} \dot{x}_{\mathrm{g}}\right)+ \\
& \quad+K\left(z_{\mathrm{a}}-1_{n \times 1} x_{\mathrm{g}}\right)+E\left(\dot{z}_{\mathrm{a}}, \dot{x}_{\mathrm{g}}\right)=0_{n \times 1},
\end{aligned}
$$

where the structure is modeled as an $n$-lumped-mass with horizontal displacement vector $z_{\mathrm{a}}(t)$ relative to an absolute (or inertial) reference, and where $x_{\mathrm{g}}(t)$ represents total ground level displacement due to the horizontal seismic motion. Notations $0_{n \times 1}, 0_{n}$ respectively, stand for column vector of dimension $n$ and matrices of size $n \times n$ with 0 as coefficients; $1_{n \times 1}$ stands for column vector with 1 as coefficients, and $1_{n}$ is the identity matrix of size $n$.

The four terms in (1) denote inertia, the damping component of the structure dynamics which includes a semiactive viscous damper located at the base level and acting solely in horizontal direction, the third term stands for the structure stiffness dynamics, and the fourth term describes the dry friction passive device. The semi-active device is assumed to be an ideal damper whose timevarying viscous damping coefficient $c_{\mathrm{A}}$ is the control input. Physically, it is bounded $c_{\mathrm{A}}(t) \in\left[0, c_{\mathrm{A}}^{\max }\right]$, with $c_{\mathrm{A}}^{\max }>0$. More complex models of semi-active actuators may be considered as, for instance, in [14] where, in addition, hysteresis and spring components are present. The dry friction passive device depicted, contributing to structure dynamics through the term $E\left(\dot{z}_{\mathrm{a}}, \dot{x}_{\mathrm{g}}\right)$ is a friction pendulum system (FPS) or bearing as often used in earthquake engineering literature. The simplest expression of the force generated by the latter device is ideally

$$
E\left(\dot{z}_{\mathrm{a}}, \dot{x}_{\mathrm{g}}\right)=\left(\begin{array}{c}
\mu N \operatorname{sign}\left(\dot{z}_{\mathrm{a} 1}-\dot{x}_{\mathrm{g}}\right) \\
0_{(n-1) \times 1}
\end{array}\right),
$$

where $\mu$ is the dry friction coefficient, $N$ is the normal force which is assumed to be constant in this work (i.e., time-variations of $N$ due to the vertical component of earthquake excitation is neglected). To cope with more realistic models, and also to avoid mathematical difficulties the sign function is often replaced by a smooth one, such as the hyperbolic tangent function.
System (1) may be put in the form

$$
M\left(\begin{array}{c}
\ddot{z}_{\mathrm{a} 1} \\
\ddot{z}_{\mathrm{a} 2} \\
\ldots \\
\ddot{z}_{\mathrm{a} n}
\end{array}\right)+C\left(\begin{array}{c}
\dot{z}_{\mathrm{a} 1} \\
\dot{z}_{\mathrm{a} 2} \\
\ldots \\
\dot{z}_{\mathrm{a} n}
\end{array}\right)+K\left(\begin{array}{c}
z_{\mathrm{a} 1} \\
z_{\mathrm{a} 2} \\
\ldots \\
z_{\mathrm{a} n}
\end{array}\right)+
$$

$$
\left(\begin{array}{c}
c_{\mathrm{A}}\left(\dot{z}_{\mathrm{a} 1}-\dot{x}_{\mathrm{g}}\right)-c_{\mathrm{b}} \dot{x}_{\mathrm{g}}-k_{\mathrm{b}} x_{\mathrm{g}}+ \\
\mu N \operatorname{sign}\left(\dot{z}_{\mathrm{a} 1}-\dot{x}_{\mathrm{g}}\right) \\
0 \\
\cdots \\
0
\end{array}\right)=\left(\begin{array}{c}
0 \\
\\
0 \\
\cdots \\
0
\end{array}\right)
$$

emphasizing the fact that both control and perturbation (seismic signal) appear only in the first equation of the system (which concerns the base level). Most often in earthquake engineering literature relative coordinates are used instead of absolute ones as done here. In addition, contrary to many previous works, the damping matrix $C$ is not assumed to be modal in this paper. Recall that modal assumption on $C$ means that the eigenvectors of the damped nominal (i.e., without uncertainty) system $(M, K, C)$ are the same as those of the undamped case $(M, K, C=0)$. The reader is referred to $[8, \S 3.5],[1$, $\S 9]$, for more details on this subject.

Specifically, when $n=2$ the structure is a 2-degreeof-freedom lumped-mass as depicted in Fig. 1 where the matrices $M, C, C_{\mathrm{c}}\left(c_{\mathrm{A}}\right)$ and $K$ are given by

$$
\begin{gathered}
M=\left(\begin{array}{cc}
m_{\mathrm{b}} & 0 \\
0 & m_{\mathrm{s}}
\end{array}\right), \quad K=\left(\begin{array}{cc}
k_{\mathrm{b}}+k_{\mathrm{s}} & -k_{\mathrm{s}} \\
-k_{\mathrm{s}} & k_{\mathrm{s}}
\end{array}\right), \\
C=\left(\begin{array}{cc}
c_{\mathrm{s}} & -c_{\mathrm{s}} \\
-c_{\mathrm{s}} & c_{\mathrm{s}}
\end{array}\right), \quad C_{\mathrm{c}}\left(c_{\mathrm{A}}\right)=\left(\begin{array}{cc}
c_{\mathrm{A}} & 0 \\
0 & 0
\end{array}\right),
\end{gathered}
$$

where all coefficients are real positive numbers.

\section{PseudoacCELERATION FLOOR RESPONSE SPECTRUM}

The objective of control design is to achieve sufficiently low values of the so-called pseudoacceleration (PSA) floor response spectrum for possible seismic disturbance $\ddot{x}_{\mathrm{g}}(t)$. In control literature, this is a perturbation attenuation problem.

For the reader who is not familiar with earthquake engineering literature the following is reported: by definition (see $\S 25.1$ of [3], Chapter 6 of [2], or $\S 7.3$ of [12] for more details), the pseudoacceleration floor response spectrum is the function

$$
(\omega, \zeta, v) \longmapsto \operatorname{PSA}(\omega, \zeta, v)=\omega^{2} \max _{t \geq t_{0}}|y(t)|
$$

where

$$
\ddot{y}+2 \zeta \omega \dot{y}+\omega^{2} y=v(t), y\left(t_{0}\right)=0, \dot{y}\left(t_{0}\right)=0,
$$

and $v(t)$ is typically the absolute acceleration of one of the $n$ floors of the structure. In this paper we are only concerned with the base level, i.e., we take $v(t)=\ddot{z}_{\mathrm{a} 1}(t)$. 


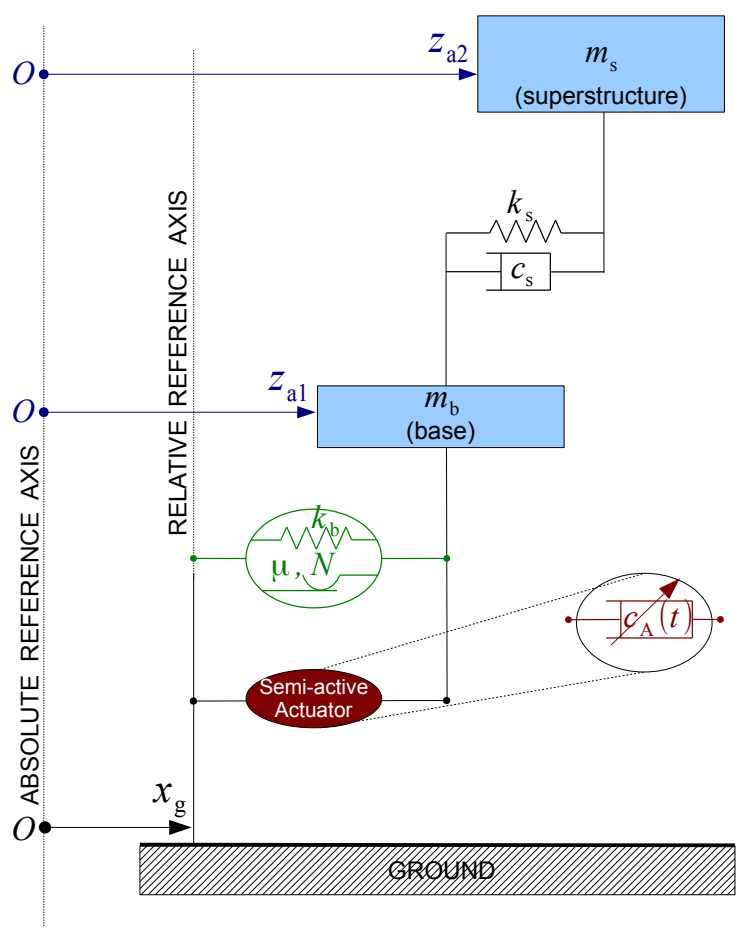

Fig. 1: A schematic diagram of a 2-degree-of-freedom structure in horizontal motion, with its absolute coordinates
Theorem 1: Consider systems described by the following type of equations

$$
\begin{aligned}
\dot{x}=(A+\Delta A(t)) x+f(x, \nu)+ & \\
& (B(x)+\Delta B(t, x, \nu)) c_{\mathrm{A}}(t)+D \nu(t),
\end{aligned}
$$

with state variable $x(t) \in \mathbb{R}^{n}$, control input $c_{\mathrm{A}}(t) \in \mathbb{R}^{m}$, and nonvanishing continuous-time perturbations $\nu(t) \in$ $\mathbb{R}^{l}$. Matrices $A$ and $D$ are known constant ones, $B$ is a known continuous function of $x$, structural uncertainties on plant model and actuator, respectively, $\Delta A$ and $\Delta B$ are unknown continuous functions of their arguments. Assume $f$ and $\rho$ to be known continuous functions of their arguments and that

(i) matrix $A$ is Hurwitz, $P$ is the unique symmetric positive-definite solution of $P A+A^{\prime} P=-Q$, given arbitrary symmetric positive definite $Q$,

(ii) functions $\nu, f, \Delta A, \Delta B$ are bounded with respective bounds $\nu^{\max }, f^{\max }, \Delta A^{\max }, \Delta B^{\max }$,

(iii) $\Delta A^{\max }<\lambda_{\min }(Q) / \lambda_{\max }(P) / 2$,

(iv) $\rho(x) \in\left[0, \rho^{\max }\right]$,

(v) $\varepsilon>0$,

(vi) and let

$$
b_{0}=\frac{\lambda_{\max }(P)}{\lambda_{\min }(P)} \frac{f^{\max }+\Delta B^{\max } \rho^{\max }+\|D\| \nu^{\max }}{\frac{1}{2} \frac{\lambda_{\min }(Q)}{\lambda_{\max }(P)}-\Delta A^{\max }} .
$$

When the signal $v(t)$ is clear from context, $\operatorname{PSA}(\omega, \zeta, v)$ is simply denoted by $\operatorname{PSA}_{\zeta}(\omega)$.

The explicit solution of $(2 b)$ is given by:

$$
y(t)=\frac{1}{\omega_{\mathrm{d}}} \int_{t_{0}}^{t} e^{-\zeta \omega(t-\tau)} \sin \left(\omega_{\mathrm{d}}(t-\tau)\right) v(\tau) \mathrm{d} \tau
$$

with $\omega_{\mathrm{d}}=\omega \sqrt{1-\zeta^{2}}$.

As is clear from its definition (2), $\operatorname{PSA}_{\zeta}(\omega)$ is not an explicit performance criterion that can be easily handled to obtain control laws. Multiple reasons can be mentioned, some of them are: (i) the non-additivity of response spectrum with respect to input signals; (ii) even for simple input signals, it is pretty difficult to construct a convex function based on $\operatorname{PSA}_{\zeta}(\omega)$ that may be used as minimization criterion. This is why in most papers dealing with this topic, closed-loop performance is expressed in terms of cost functions based on maximum displacement, velocity and acceleration of the structure in response to a number of given seismic signals [13].

In the present work a better insight to an explicit use of response spectrum as criterion is proposed.

\section{A Robust CONTROL DESIGN THEOREM}

The following result is largely inspired by the work of G. Leitmann and co-authors, see for instance [9], [4], [11], $[6],[7]$.
If the control input is set as follows

$$
\begin{aligned}
& c_{\mathrm{A}}(x)=\max \left(0_{m \times 1}, p(x)\right) \\
& p(x)= \begin{cases}-\frac{B(x)^{\prime} P x}{\left\|B(x)^{\prime} P x\right\|} \rho(x), & \left\|B(x)^{\prime} P x\right\| \geq \varepsilon, \\
-\frac{B(x)^{\prime} P x}{\varepsilon} \rho(x), & \left\|B(x)^{\prime} P x\right\|<\varepsilon,\end{cases}
\end{aligned}
$$

then all closed-loop trajectories $x(t)$ are bounded, and, for any initial conditions $x_{0}$ satisfying $\sqrt{x_{0}^{\prime} P x_{0}}>$ $\sqrt{\lambda_{\min }(P)} b_{0}$, and for all $b>b_{0}$ the trajectories $x(t)$ are driven into balls of radius $b$ within time

$$
T=\frac{\ln \left(\frac{\sqrt{x_{0}^{\prime} P x_{0}}-\sqrt{\lambda_{\min }(P)} b_{0}}{\sqrt{\lambda_{\min }(P)}\left(b-b_{0}\right)}\right)}{\frac{1}{2} \frac{\lambda_{\min }(Q)}{\lambda_{\max }(P)}-\Delta A^{\max }} .
$$

The proof of this theorem is not included here for lack of space. It was constructed using uniform boundedness and uniform ultimate boundedness tools as in Theorem 4.18 of [10] and page 1141 of [4].

The following are comments on the differences between Thm 1 and its inspiring results in [9]. In [9], [4], [11] matching conditions provide the means to dominate the cumulative effect of perturbations and structured uncertainties on plant model and actuator, by using a sufficiently strong active control law (7). In [9], [4], [11], [7] when $\varepsilon=0$ in (7) and the lower branch of control law is removed it can be shown that global asymptotic 
stability (GAS) of origin can be ensured. This means that the energy dissipation mechanism is ensured for all bounded perturbation signals, and for all $t \geq 0$.

The proposed SAC in Thm. 1 is not designed to ensure GAS of origin in any particular situation. Actually, hypotheses of Thm. 1 are not sufficient to prove GAS of origin. The control design function $\rho(x)$ can be chosen arbitrarily small in Thm. 1 which is not the case in [9], [4], $[11],[7]$ where a necessary lower bound, $\rho(x) \geq \rho^{\text {min }}>$ 0 is calculated based on maximum amplitude value of perturbation. This allowed us to freely tune the function $\rho(x)$ in order to attain performance criterion like floor response pseudoacceleration spectrum.

It is worth noting that the control (6) is a continuous function of variable $x$.

Summarizing, the theorem provides this very interesting design tool: application of the control allows to bring system trajectories into bounds $b$ within time $T$ as long as $b>b_{0}$ where the limiting bound $b_{0}$ is of course a pretty complex function of model uncertainties and control design parameters. In addition the theorem says that the higher the ratio $\lambda_{\min }(Q) / \lambda_{\max }(P)$ is the higher amount of uncertainty $\Delta A$ can be tolerated. However, clearly, the desirable minimization of $b_{0}$ and $T$ with respect to control design parameters is not simple given the complexity of the dependance of $b_{0}$ and $T$ on model uncertainties and control parameters.

Applying Thm. 1 to the structure model (1) we show the following

Corollary 2: The floor response spectrum evaluated at the base level is uniformly bounded over the entire frequency range:

$$
\operatorname{PSA}_{\zeta}(\omega) \leq \frac{1}{m_{\mathrm{b}}} \frac{1}{\zeta \sqrt{1-\zeta^{2}}}\left\|v_{1}\right\| d\left(\left\|x_{0}\right\|\right)
$$

where

$$
\begin{gathered}
v_{1}^{\prime}:=\left(-\left(k_{\mathrm{b}}+k_{\mathrm{s}}\right) \quad k_{\mathrm{s}}-\left(c_{\mathrm{b}}+c_{\mathrm{s}}+c_{\mathrm{A}}^{\max }\right) \quad c_{\mathrm{s}}\right), \\
d\left(\left\|x_{0}\right\|\right)= \begin{cases}\sqrt{\frac{\lambda_{\max }(P)}{\lambda_{\min }(P)}}\left\|x_{0}\right\|, & \left\|x_{0}\right\| \geq \mu_{0}, \\
\mu_{0}, & \left\|x_{0}\right\|<\mu_{0},\end{cases}
\end{gathered}
$$

with

$$
\begin{gathered}
\mu_{0}=\frac{\frac{\mu N}{m_{\mathrm{b}}}+\sqrt{n} \ddot{x}_{\mathrm{g}}^{\max }}{\frac{1}{2} \frac{\lambda_{\min }(Q)}{\lambda_{\max }(P)}-\Delta A^{\max }}, \\
\ddot{x}_{\mathrm{g}}^{\max }=\max _{t \geq 0}\left|\ddot{x}_{\mathrm{g}}(t)\right| .
\end{gathered}
$$

This corollary is not the best wanted result in practice: a better bound would be in terms of frequency $\omega$ showing how the floor response peaks are decreased at specific frequencies. Nevertheless it seems to be the first result linking floor response spectrum to control design parameters.

\section{SEMI-ACTIVE CONTROL}

As already mentioned it is a standard practice in earthquake engineering (see [8]) to use real-valued modal coordinates as follows.

Let $\left(\omega_{i}^{2}, \varphi_{i}\right), i=1, \ldots, n$ be the couples of eigenvalues and right eigenvectors associated with the symmetric matrices $M$ and $K$, amd $M$ is positive-definite: for each $i, \omega_{i}$ and $\varphi_{i}$ verify

$$
K \varphi_{i}=\omega_{i}^{2} M \varphi_{i}, \quad \varphi_{i}{ }^{\prime} M \varphi_{j}=\delta_{i j}, \quad \varphi_{i}{ }^{\prime} K \varphi_{j}=\omega_{i}^{2} \delta_{i j},
$$

where $\delta_{i j}$ is the Kronecker symbol. $\omega_{i}$ and coefficients of $\varphi_{i}$ are all real-valued. Let $\left(\omega_{i}^{2}, \varphi_{i}\right)$ be numbered such that $\omega_{1}^{2} \leq \omega_{2}^{2} \leq \cdots \leq \omega_{n}^{2}$. The reader may refer to $[5, \S 11]$ for more details on the algebra of symmetric positive definite generalized eigenvalue problem. The eigenvectors $\varphi_{i}$ are linearly independent [5, Thm. 11.9] and therefore form a basis; they are referred to as the modes of vibration [8]. Let

$$
\phi=\left(\begin{array}{llll}
\varphi_{1} & \varphi_{2} & \cdots & \varphi_{n}
\end{array}\right)
$$

be the matrix of modal shapes, so that (11) can be rewritten in matrix form as:

$$
K \phi=M \phi \Lambda, \quad \phi^{\prime} M \phi=1_{n}, \quad \phi^{\prime} K \phi=\Lambda,
$$

with

$$
\Lambda=\operatorname{diag}\left(\omega_{1}^{2}, \omega_{2}^{2}, \ldots, \omega_{n}^{2}\right) .
$$

Let $q$ be the modal coordinates vector defined such that

$$
z_{\mathrm{a}}(t)=\phi q(t)=\varphi_{1} q_{1}(t)+\varphi_{2} q_{2}(t)+\cdots+\varphi_{n} q_{n}(t) .
$$

Though not limited to two-degree-of-freedom structures, this semi-active control is applied to the case $n=2$ for simplicity in the sequel. In other words, instead of giving the method in its full generality, let us explain it on a simple example.

A. Choice of $Q$ : Modal coordinates transformations are used in the following way to influence the floor response spectrum. Let $W$ be a negative definite function,

$$
\begin{aligned}
W & =-\gamma_{1} q_{1}^{2}-\gamma_{2} q_{2}^{2}-\gamma_{3} \dot{q}_{1}^{2}-\gamma_{4} \dot{q}_{2}^{2} \\
& =-\left(\begin{array}{ll}
q^{\prime} & \dot{q}^{\prime}
\end{array}\right) \operatorname{diag}\left(\gamma_{1}, \gamma_{2}, \gamma_{3}, \gamma_{4}\right)\left(\begin{array}{c}
q \\
\dot{q}
\end{array}\right),
\end{aligned}
$$

with positive real parameters $\gamma_{i}>0$. Parameters $\gamma_{1}$ and $\gamma_{3}$ are used to penalize the effect of first vibrational mode, in terms of generalized modal displacement and velocity, respectively. Similarly $\gamma_{2}$ and $\gamma_{4}$ are used to penalize the effect of second vibrational mode.

One can use transformation (14) in order to express $W$ from (15) in terms of absolute coordinates vector $x$ with

$$
x=\left(\begin{array}{c}
z_{\mathrm{a}} \\
\dot{z}_{\mathrm{a}}
\end{array}\right),
$$

By setting $-x^{\prime} Q x=W$ and using the last two biorthogonality properties from (13) in order to calculate explicitly 
the inverse of matrix $\phi$ from (12), direct calculation leads to the following bloc diagonal matrix

$$
\begin{aligned}
Q=\operatorname{diag}\left(M^{\prime}\left(\gamma_{1} \varphi_{1} \varphi_{1}{ }^{\prime}+\gamma_{2} \varphi_{2} \varphi_{2}{ }^{\prime}\right) M,\right. \\
\left.M^{\prime}\left(\gamma_{3} \varphi_{1} \varphi_{1}{ }^{\prime}+\gamma_{4} \varphi_{2} \varphi_{2}{ }^{\prime}\right) M\right),
\end{aligned}
$$

and the structure model (1) can be rewritten as

$$
\dot{x}=A x+f(x, \nu)+(B(x)+\Delta B(\nu)) c_{\mathrm{A}}+D \nu .
$$

where

$$
\begin{aligned}
& A=\left(\begin{array}{cc}
0_{2} & 1_{2} \\
-M^{-1} K & -M^{-1} C
\end{array}\right), \Delta B(\nu)=\left(\begin{array}{c}
0 \\
0 \\
\dot{x}_{\mathrm{g}} / m_{\mathrm{b}} \\
0
\end{array}\right), \\
& f(x, \nu)=\left(\begin{array}{c}
0_{2 \times 1} \\
-M^{-1} E(x, \nu)
\end{array}\right), B(x)=\left(\begin{array}{c}
0_{2 \times 1} \\
-\dot{z}_{\mathrm{a} 1} / m_{\mathrm{b}} \\
0
\end{array}\right) \text {, } \\
& D=\left(\begin{array}{c}
0_{2} \\
M^{-1} K 1_{2 \times 1}
\end{array}\right) \text {, and } \nu=\left(\begin{array}{c}
x_{\mathrm{g}} \\
\dot{x}_{\mathrm{g}}
\end{array}\right) .
\end{aligned}
$$

The initial condition of (18) is $x(0)=0_{2 n \times 1}$.

B. Choice of $\rho(\cdot)$ : In order to be able to protect the actuator from damage, the maximum allowed damping force $F^{\max }$ can be taken into account in the design of control law parameters by setting

$$
\rho\left(\dot{z}_{\mathrm{r} 1}\right)=\max \left\{\rho^{\max }, F^{\max } /\left|\dot{z}_{\mathrm{r} 1}\right|\right\},
$$

where the relative velocity at the base level $\dot{z}_{\mathrm{r} 1}=\dot{z}_{\mathrm{a} 1}-\dot{x}_{\mathrm{g}}$.

System (18) is of the form (4) allowing to apply Thm 1.

\section{Simulation RESUlts}

In this section we illustrate the performance of the proposed semi-active control through the 2-degree of freedom structure already detailed throughout the previous section. The parameters (in consistent units) which are used in the simulations are as follows: $m_{\mathrm{b}}=0.25$; $m_{\mathrm{s}}=1 ; k_{\mathrm{b}}=12.3370 ; c_{\mathrm{s}}=1.8850 ; k_{\mathrm{s}}=355.3058 ;$ $c_{\mathrm{A}}^{\max }=1.5708 ; \sigma(y)=\tanh (11 \times y)$. The floor response spectra are calculated for $\zeta=2 \%$ damping.

Multiple control scenarios are shown for comparison:

- NC (no control): this is the uncontrolled structure dynamic response to the seismic solicitation. Specifically, the structure is seismically isolated by a dry friction device with a low value for friction coefficient $\mu$. In the simulations to be shown we used $\mu=7.48 \times 10^{-3}$.

- PC (passive control): an additional damping is put at the base level consisting of a high damping rubber bearing (HDRB), so that the total equivalent damping coefficient is $\xi_{\mathrm{b}}+\Delta \xi_{\mathrm{b}}=25 \%$. In simple words, in Fig. 1 the friction device is removed and replaced by a damper with damping coefficient $c_{\text {tot }}=2.2777$.

- SAC corresponds to implementing control law (6) on plant model (1) or (18), with adjustment parameters $\varepsilon=5 \times 10^{-3}, \gamma_{1}=\gamma_{3}=5 \times 10^{-4} ; \gamma_{2}=5 \times 10^{2} ;$ $\gamma_{4}=10^{-5} ; \rho(\cdot) \equiv c_{\mathrm{A}}^{\max }$.

A. Choice of seismic records: Instead of real records of seismic signal it was found more illustrative to use artificial ones for $\ddot{x}_{\mathrm{g}}(t)$. These signals suit the ground response pseudoacceleration spectrum specification of Cadarache rock site, in southern France. They were scaled in amplitude to reach a maximum absolute value of $0.6 g$, with $g=9.81 \mathrm{~m} / \mathrm{s}^{2}$, so that they correspond to strong motion earthquake accelerograms. Actually, it was our intention to use signals that ensure control to be within the full range of 0 to $c_{\mathrm{A}}^{\max }$. Otherwise, if signals are too weak compared to a reference, namely the signal used to calibrate control parameters, the effect of adding a SAC force is negligible with respect to the natural behavior of structure. On the contrary, if seismic signals are too strong the SAC may saturate and it will act as PC with high damping, which again is not wanted. In other words, prior to implementing in practice this $\mathrm{SAC}$, it is necessary to have some a priori knowledge on the maximum amplitude of seismic signal to hit the structure. In earthquake engineering, this information is often used in seismic characterization and is called peak ground acceleration, with the definition (10). On the other hand, it is also required by Thm. 1 as in its second assumption.

Moreover, the selection of seismic signals has been made such that they are wide-band sufficiently rich in

- low spectral content around first eigenfrequency so that we can notice a pretty large first peak on floor response spectrum curve when using $\mathrm{NC}$,

- higher spectral content around second eigenfrequency, otherwise, phenomena related to amplification of second mode of vibration, e.g., when using PC, might not be visible in terms of floor response spectrum. Often, this is the case for earthquake signals recorded on stiff soil and rock sites.

B. Calibration of control law parameters: The control parameters are calibrated once for all three simulations, using a trial and error approach. They are calculated with the seismic signal used to draw results of Fig. 2. The other two figures then serve for validation and to support further discussions on control capabilities.

C. Interpretation: Since the choice of control parameters has been made with respect to only one seismic signal, one may wonder whether or not the result concerning adjustment control parameters is globally available. By looking at Figs. 3-4, one can notice a visible advantage and improvement when using SAC over PC in terms of floor response spectrum evaluation especially around second eigenfrequency. Actually, the PC curve is used in this work as a qualitative indicator of a worst case behavior for validating the use of more complex $\mathrm{SAC}$ techniques over the simpler passive control. In other words, if there is no visible improvement of $\mathrm{SAC}$ over $\mathrm{PC}$, the SAC should be disregarded since there is no 
practical interest in using it. Fortunately, our simulations show that SAC is doing better than PC, at the expense of increased implementation cost. Extended simulations with randomly chosen natural, historical, seismic events showed that, as a worst case behavior of this SAC device with fixed adjustment parameters, the performance in terms of floor response spectra will be at least as good as PC.

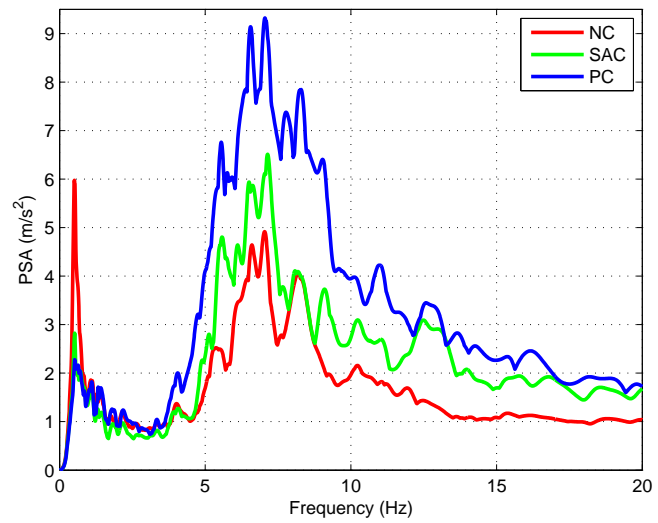

Fig. 2: Floor response spectrum at the base in response to seismic signal record no. 1

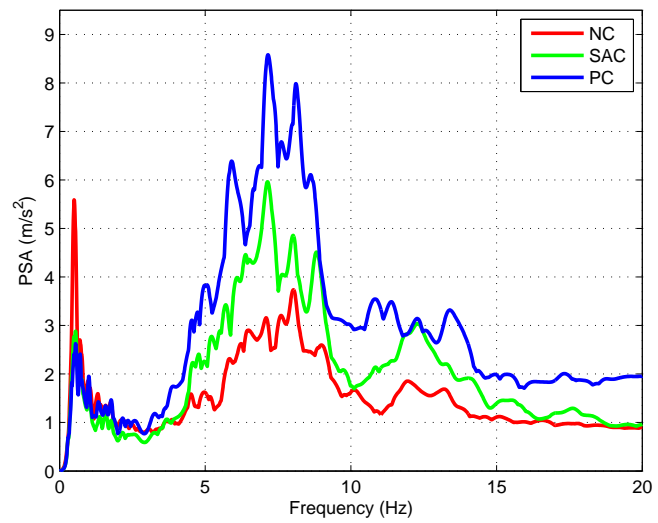

Fig. 3: Floor response spectrum at the base in response to seismic signal record no. 2

\section{CONCLUSIONS}

In this paper Leitmann and co-authors's results on the stabilization of uncertain nonlinear systems and on earthquake structure protection both have been revisited. Bounds are calculated guaranteeing some interesting control design parameters. This result is more user friendly. The major achievement lies in the adaptation of control law and system dynamics towards solving SAC problems. A second contribution of this paper consisted in presenting a method for choosing SAC parameters, based on vibrational modes analysis. One reason for proceeding in this direction is that performance in closedloop of structure response to unknown seismic signals is evaluated qualitatively in terms of floor response pseudoacceleration spectrum. We showed that working with information related to balls radius size in state space coordinates might provide efficient and possible means to reduce the floor response spectral bound.

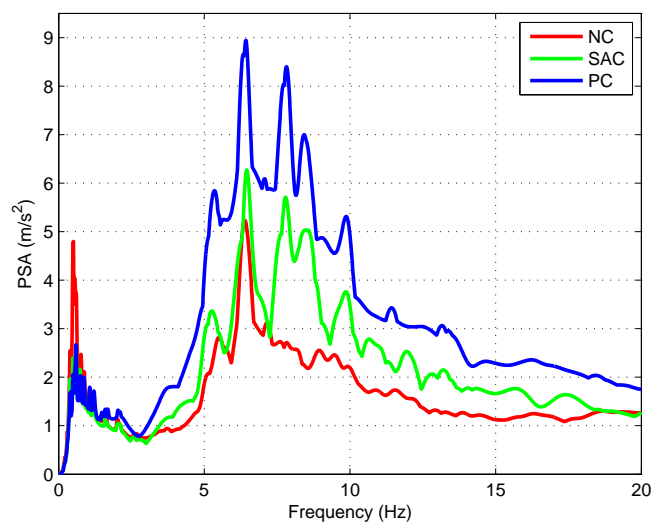

Fig. 4: Floor response spectrum at the base in response to seismic signal record no. 3

\section{REFERENCES}

[1] J.H. Argyris, H.P. Mlejnek, Dynamics of structures, Texts on computational mechanics, North-Holland, Amsterdam, The Netherlands, 1991.

[2] A.K. Chopra, Dynamics of Structures: Theory and Applications to Earthquake Engineering, Third edition, Prentice Hall, Upper Saddle River, NJ, 2007.

[3] R.W. Clough, J. Penzien, Dynamics of Structures, Third edition, CSI, Berkeley, CA, 2003.

[4] M.J. Corless, G. Leitmann, Continuous State Feedback Guaranteeing Uniform Ultimate Boundedness for Uncertain Dynamic Systems, IEEE Trans. Automat. Contr., AC-26(1981), 1139-1144.

[5] B.N. Datta, Numerical linear algebra and applications, second edition, SIAM, Philadelphia, PA, 2010.

[6] S. Gutman, Uncertain dynamical systems-A Lyapunov minmax approach, IEEE Trans. Automat. Contr., 24(1979), 437443.

[7] S. Gutman, G. Leitmann, Stabilizing feedback control for dynamical systems with bounded uncertainty, in Proc. IEEE Conf. Decison Contr., 1976, pp. 94-99.

[8] D.J. Inman, Vibration with Control, John Wiley \& Sons, West Sussex, England, 2006.

[9] J.M. Kelly, G. Leitmann, A.G. Soldatos, Robust control of base-isolated structures under earthquake excitation, $J$. Optimization Theory and Applications, 53(1987), 159-180.

[10] H.K. Khalil, Nonlinear Systems, Third edition, Prentice-Hall, Upper Saddle River, NJ, 2002.

[11] G. Leitmann, Guaranteed Asymptotic Stability for a Class of Uncertain Linear Dynamical Systems, J. Optimization Theory and Applications, 27(1979), 99-106.

[12] F. Naeim, J.M. Kelly, Design of Seismic Isolated Structures: From Theory to Practice, John Wiley \& Sons, New York, 1999.

[13] S. Narasimhan, S. Nagarajaiah, E.A. Johnson, Smart baseisolated benchmark building part IV: Phase II sample controllers for nonlinear isolation systems, Struct. Control Health Monit., 15(2008), 657-672.

[14] I. Politopoulos, H.K. Pham, Floor spectra of mixed base isolated structures, Bull. Earthquake Engng., 9(2011), 11151135 . 